\title{
An Elementary Communication Framework for Open Co-operative RoboCup Soccer Teams
}

\author{
Luís Mota ${ }^{1,2}$ and Luís Paulo Reis ${ }^{2}$ \\ 1 DCTI-Instituto Superior de Ciências do Trabalho e da Empresa, Av. das Forças \\ Armadas 1649-026 Lisboa, Portugal luis.mota@iscte.pt \\ 2 LIACC-Faculdade de Engenharia da Universidade do Porto, Rua Dr. Roberto Frias \\ s/n 4200-465 Porto, Portugal
}

\begin{abstract}
One of the present day challenges in RoboCup is the development of Open Co-operative teams, where different research labs join efforts to build a common team. In RoboCup Middle-size League, some teams were confronted with a pairwise cooperation scenario in order to qualify to the competition. Such teams bring together robots with heterogeneous hardware, architectures and control software, which hinders straightforward co-operation.

The robots in these teams might co-operate through a-priori strategic knowledge and structured communication during the game. This paper presents the kernel of a communication framework, defining a robotic soccer vocabulary, as well as rules to manage communication.
\end{abstract}

\section{Introduction}

RoboCup ${ }^{3}$ is an international initiative to promote Artificial Intelligence, robotics, and related fields. It fosters research by providing a standard problem where a wide range of technologies can be integrated and examined. RoboCup uses the soccer game as a domain of research, focusing on topics such as multi-agent collaboration, real-time reasoning, robotics, and sensor-fusion. The ultimate goal of the RoboCup initiative is "By the year 2050, develop a team of fully autonomous humanoid robots that can win against the human world soccer champion team." What kind of robotic team will play this decisive game? How will this team be selected? What will the team's high-level architecture be?

This team will surely be formed by heterogeneous robots, originating from distinct research labs, a selection of the best players, which will outperform any single-origin team, such as it is the case in human soccer. If this is to be the case, how will such a team be built and managed, and how will it play?

Recently, in the Middle size league, the Technical Comittee has decided that some of the teams will have to join efforts to pairwise create common teams. This means that the question of building multi-partner teams has to be dealt with in the near feature. Moreover, this challenge had previously been put forward after the RoboCup 2004 by it's chairs. In the 4-legged league, this issue has

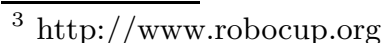


also been dealt with, namely by the German Team [1], which, using a common architecture jointly developed by four different partners, has won the RoboCup championship in 2004 and 2005.

This subject has recently been the subject of a prospective analysis [2], through the design of two application scenarios where communication is the basis for co-operation in open co-operative teams. In the present paper, a Communication Framework that leads to implementing these scenarios is defined. In section 2, a simplified scenario that reminds this work's motivation is outlined. To fulfil this scenario, there will be the need for a vocabulary relative to robotic soccer, presented in section 3 . The management of interactions between players during the game must also be determined, and a proposal is made in section 4 . Finally, we present a summary and look into future work in section 5 .

\section{Initial co-operation scenario}

In a hypothetical situation where two or more teams join efforts to create a single team, co-operation will undoubtedly be a difficult problem to solve, since each team has designed their robots differently and set up distinct communication, playing and positioning strategies. In a first phase, the joint team should therefore be expected to play straightforwardly, avoiding complicated set moves and trying to keep its' game simple. The framework presented in the remainder of this paper aims at supplying this scenario's needs, which is a simplification of [2].

In the first games played by the joint team, the line-up will be decided by some human coach, that will choose the strategy and which players should play in each position. This information will simply be transmitted to the players. Nevertheless, some information about each player should also be available, e.g the reach and precision of their kicking devices. Such information can be used during the game to support decisions.

Surely, the most basic co-operative task is to allow players to exchange information regarding the state of the game. As players extract data from their sensors, they should transmit it to their team-mates, contributing to the update of the individual world models. The exchanged information should include players, ball and referee position. Other relevant events, such as a one player being sent off, should also be reported.

Communication can also be used by the players to co-operate further. Namely, a player may consider that, in order to score a goal, it needs another robot to perform some action, like passing the ball or moving to a specific area of the playing field. Also, when a player decides to take some action, it can announce it to its' team members, and these will be able, e.g, to change their positioning accordingly.

Another possibility is that, during the game, the team needs to change the strategy, because the desired results are not being reached. In such a situation, after the strategy change is decided, it should be broadcasted to all team members. 


\section{Robotic soccer domain concepts}

\subsection{Physical objects}

As pointed out in the scenario presented in section 2, robots, to co-operate, will have to share information about the world state, obtained through their sensors. They will therefore have to use a vocabulary describing the objects on the soccer terrain. The most important of these objects are the players, both team-mates and opponent players. They can all be modelled similarly, being identified by some string, player number and being labelled with their team name. The subclasses 'OwnTeamPlayer' and 'OpponentTeamPlayer' were created because during the game it is difficult to precisely identify a player, but it may be possible to recognise it as being from the own team or from the opponent. In such cases, the team can be distinguished without having to identify the player.

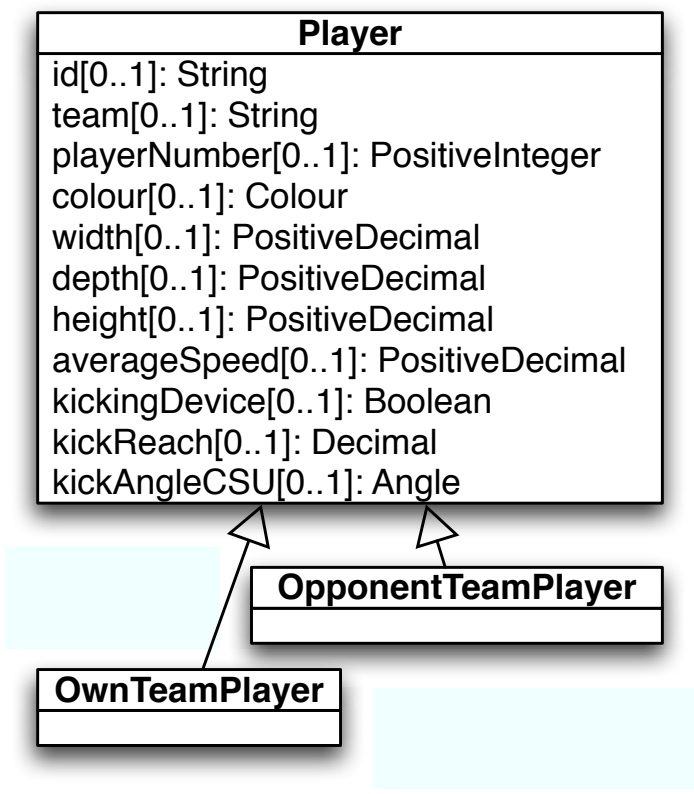

Fig. 1. Player definition

To assist the robots' visual recognition, information about their colour and shape (width, height and depth) should also be expressable. Additionally, they should be characterised by their skills, allowing team-mates to consider these skills when deciding how to play. Relevant skills are their average motion speed, the existence of a kicking device and its reach and precision. This modelling, where all attributes are optional, because information may be unavailable, can 
be seen as an UML diagram in figure 1. The kicking precision will be made clear in the next section, when we introduce uncertainty related concepts.

Other relevant physical objects are the ball and the referee, which are persistently present on the soccer field. The ball should be characterised by its' colour and radius, to enable its' recognition during the game. The referee should have his visual aspects represented, such as his height and clothing colour, for the same reasons. The modelling of these concepts, as well as the layout of the whole physical objects hierarchy, can be seen on figure 2 .

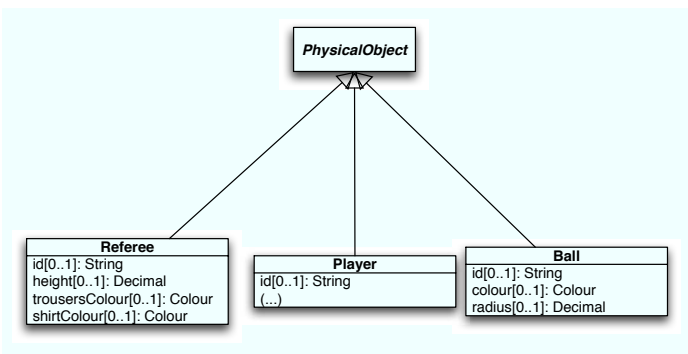

Fig. 2. Physical Objects

\subsection{Object positioning}

Positioning of objects is also of vital importance during the game. As players extract information from sensor data analysis, they should send it to their teammates, in order to contribute to their view of the state of the world. The robots will need therefore to be able to express the positioning of the dynamic objects.

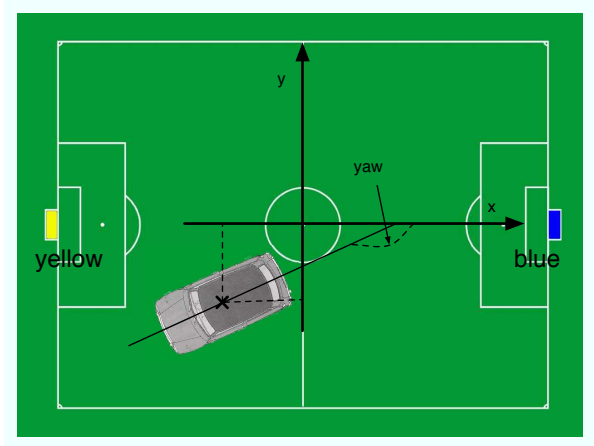

Fig. 3. Placement of axis on the field: z-axis points upwards and is not shown. 
The absolute pose of a player (or other object) is based on a right-hand cartesian co-ordinate system, with the origin placed at the centre of the field, the $\mathrm{x}$-axis pointing at the blue goal and the $\mathrm{z}$-axis up, as shown in figure 3 . Naturally, while using present day robots, the z-co-ordinate will be zero, since they are permanently on the ground. On its turn, the ball may have a non-zero z-co-ordinate.

The robot's orientation, i.e., the direction it is facing, is also represented in figure 3, where the robot is pictured as a car for clarity. The robot's only possible rotation, around the $\mathrm{z}$-axis, can be modelled as a yaw angle relative to the $\mathrm{x}$ -

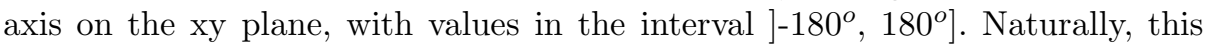
yaw value has no meaning for the ball, and should be left blank. The definition of 'AbsolutePositioning' can be found in figure 4 .

In terms of positioning, another issue must be considered: the uncertainty in the determinations made by the robots, which are placed in a dynamic environment and deal with faulty and inaccurate sensing data. In fact, no measurement is entirely reliable and, as argued by [3], different sensors introduce different kinds of uncertainty. As an example, a laser range finder has a good accuracy for distance, but is of no use to identify objects. A camera is very useful to identify objects, gets good results when determining relative angles, but has a high uncertainty regarding distance. Therefore, there should be a way of expressing uncertainty in the different components of an observation. We chose to use the Standard Uncertainty ${ }^{4}$, as defined in [4], which is a well known standard definition. A class (AbsolutePositioning WithSU, for Standard Uncertainty) was added, which extends 'AbsolutePositioning' and introduces attributes to estimate the uncertainty for each of the components of the positioning. Naturally, there can also be uncertainty about the identity of the observed object. For example, a robot might see another robot but be unsure about its identity. To model this level of confidence, the 'targetIdentification Confidence' attribute was defined.

When one considers attentively the scenario in section 2 , a simple observation arises: most of the positioning exchanged are determined by a robot. All measurements are made from the viewpoint of the robot, and are thus relative to the robot's position. Additionally, the prime sensor used in these observations will be a camera, which will accurately estimate the relative angular difference to the other objects, but that will have a considerable uncertainty when determining the distance to the same objects. Considering these factors, and with the goal of keeping the uncertainty about own-positioning independent from the estimation of other objects' position, there is the class (RelativePositioning), representing relative positioning with respect to the observer. This class uses polar co-ordinates and refers to the observer by its identification (observerID).

\footnotetext{
${ }^{4}$ If the measurement value is $\mathrm{y}$, the probability distribution of the measurement is approximately normal (Gaussian) and the Standard Uncertainty $\left.\left(u_{(} y\right)\right)$ is well estimated, then the interval $\left.\left.\left[y-u_{(} y\right), y+u_{(} y\right)\right]$ is expected to encompass approximately $68 \%$ of the distribution of values that could reasonably be attributed to the value of the quantity $\mathrm{Y}$ of which $\mathrm{y}$ is an estimate.
} 
Naturally, the class 'RelativePositioningWithSU' extends the former to include information about the uncertainty.

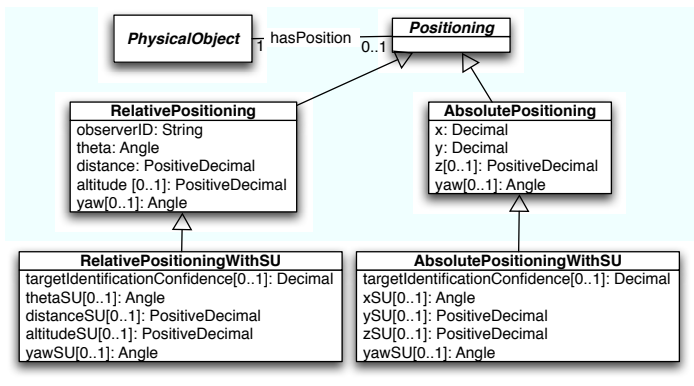

Fig. 4. Positioning related concepts

The next section needs the definition of field regions, which are also positioningrelated. [5] introduce a definition of such regions, including predefined areas such as 'our_penalty_box', as well as freely definable areas like circles. These region definitions will therefore be included in this framework.

\subsection{Game events}

During the game, some events occur and may be reported to team-mates, since they are relevant to the world state. Such events are related to temporarily absent players, which may influence decisions or even strategy changes. These events are:

sentOff(player) the player has been sent off by the referee;

returnedToGame(player) the player has returned to the game, after having been sent off;

malfunctioning(player) the player is immobilised in a position in the field and seems therefore to be malfunctioning;

functioning(player) the player seems to be functioning normally.

\subsection{Player moves and actions}

The players are not limited to exchanging information: co-operation can be enhanced by the intentional exchange of messages to co-ordinate robots' behaviour. One example, from [6], is when a robot well positioned to score a goal decides to ask its team-mate holding the ball to perform a pass. Such interactions rely on the prior existence of a vocabulary defining skills and actions, which can, during the game, be requested.

[5] define a set of actions, which will be used in the present framework. Some of these actions have added arguments, to better characterise their application. These actions and moves are: 
shoot() shoot the ball at the opposite goal;

pass(playerID) pass the ball at the team-mate identified by playerID;

forward(fieldRegion) pass the ball to the region identified by fieldRegion;

dribble(direction) dribble while moving in some direction, defined as an angle

with the $\mathrm{x}$-axis in the range $\left.]-180^{\circ}, 180^{\circ}\right]$;

run(direction) run (with or without holding the ball) in some direction;

hold() keep the ball;

clear () throw the ball deliberately outside;

intercept () intercept the ball, which is being held by the opponent;

tackle(playerID) steal the ball from the opponent identified by playerID;

mark(playerID) mark individually the player identified by playerID;

markPassLine(playerID1, playerID2) mark the pass line between the play-

ers identified by playerID1 and playerID2;

gotoBall() go to the ball position, and

move(fieldRegion) move to the region identified by fieldRegion.

\subsection{Tactics definitions}

Tactics are a complex soccer concept, defining the players' preferred positioning on the field (formations), as well as the game pace and the team's pressure and mentality. These definitions will influence the players' options, and also their choice of actions. During a game, when the expected results are not being reached, a tactics change may be necessary, and therefore decided by the team. Such a change will only have effect if it can be communicated to all the players, and thus there must be a way of expressing it. A set of classes has been defined and can be seen in figure 5 .

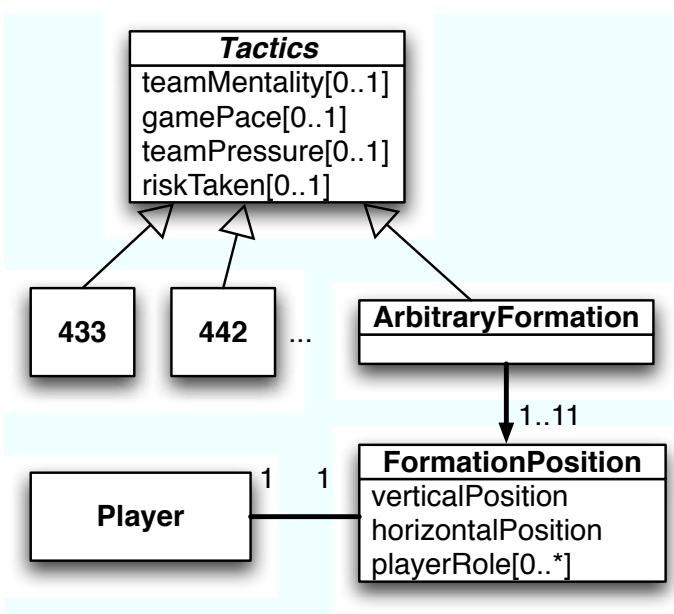

Fig. 5. Tactics related concepts 
Most of the attributes in the 'Tactics' class have a discrete set of possible values, which will be looked into now. teamMentality will take values from veryDefensive to veryOffensive. gamePace intends to represent the speed imposed by the team, and can have an integer value from 0 to 100 . teamPressure is the kind of pressure exercised, with values from veryLow to veryHigh. riskTaken is a concept which will be used, e.g., when a team is losing the game and the time is running out: the risk taken will be higher, with values from 0 to 100 .

The relative positioning of the players on the field is also of great importance. There are predefined formations, like the ones presented as examples in figure 5: 442 and 433. Nevertheless, in particular situations, there may be the need to use out-of-the-ordinary formations, which will be arbitrarily defined. Such formations will make use of the ArbitraryFormation class, that will have up to eleven different allocations of players to specific positions, as represented by the FormationPosition class. In this class, the positioning of each player is characterised by an horizontal position (with possible values left, leftCentre, centre, rightCentre and right) and a vertical position (with values ranging from sweeper to forward). The attribute playerRole will define the attitude of the player and can have multiple values from the set normal, shooter, dribbler, passer, positional and marker. The two latter values concern the behaviour while defending, while the former are attack-related.

\section{Inter-robot communicative interactions}

The previous section has defined an information schema modelling the fundamental concepts in robotic soccer. Since this information is to be shared between heterogeneous agents, one also needs to establish how this exchange will be managed, to ensure that the interactions between robots are conveniently understood. The autonomous agents' community has been dealing with these problems for several years, and one can therefore profit from the results previously obtained.

The transmission of observed information, including positioning of objects or game events, needs only a simple interaction, where one player (Sender) will inform some other players (Receivers). The Receivers may optionally send an acknowledgement back to the Sender. This interaction protocol is represented as an AUML diagram ${ }^{5}$.

This protocol will also be used to advertise choices: when a player has decided to do a specific action, it sends this information to its team-mates. An example of a message, where a robot informs others that it intends to shoot at the opposite goal, uses one of the actions defined in section 3.4 and the intends $(I)$ operator defined in [7], and is as follows:

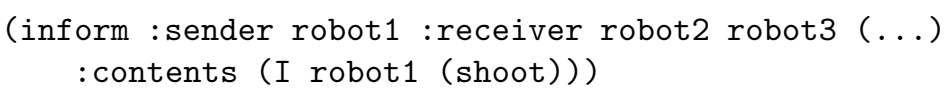

Other interactions are slightly more complex: if a player wants a team-mate to perform a specific action, it will have to request this action, and the requested

\footnotetext{
5 http://www.auml.org
} 


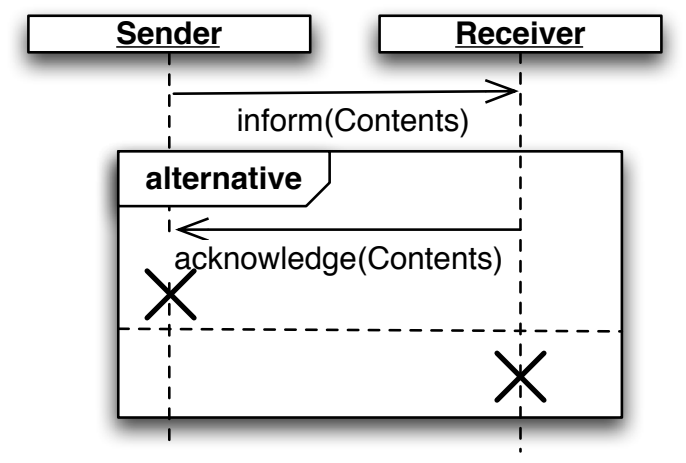

Fig. 6. Inform interaction protocol

player will have to either accept or reject the request. Such an interaction resembles the FIPA Request Protocol, defined in [8], which has been simplified to the interaction displayed in figure 7 . According to this protocol, the Sender will request the Receiver to perform one of the actions defined in section 3.4. The Receiver will then reply accepting or rejecting the request.

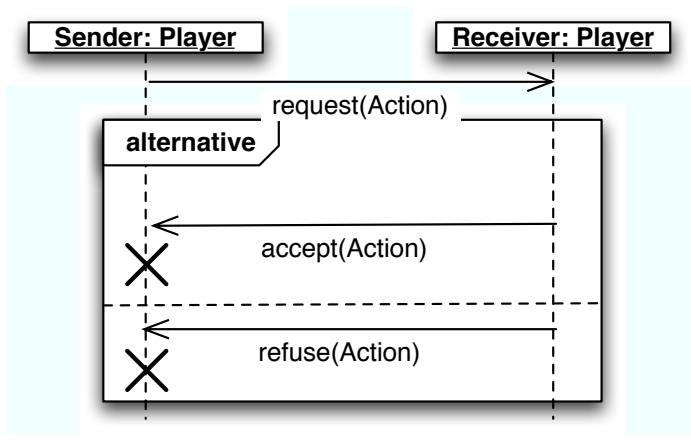

Fig. 7. Request interaction protocol

\section{Summary, conclusions and future work}

A communication framework has been defined, contributing to the development of joint, multi-partner, heterogeneous, co-operative and open RoboCup soccer teams. This framework introduces, a vocabulary defining a fundamental set of concepts needed by robots to communicate during a match. This vocabulary includes the definition of physical objects, such as robots, the ball and referees. The positioning of these objects was also modelled, as well as the underlying 
uncertainty when obtained from sensor readings. The vocabulary also defines game events, e.g., a player being sent off the game. In terms of game playing, individual moves (shoot, dribble, clear,...) have been defined, and can be used to compose complex interactions between players. On the team level, there is a definition for tactics, with concepts as formations, team mentality and risk taken. In short, this vocabulary delivers the grounding of the concepts which heterogeneous robots can use to co-operate through communication.

The existence of a shared vocabulary is, however, not enough for robots to communicate. They also have to agree on the management of the interactions between them. With this purpose, two kinds of interactions have been defined. The first kind allows robots to share information about the game and their individual intentions. The second enables momentary co-operation that will lead to more complex moves involving several robots.

This framework is therefore a fundamental set of concepts and protocols for robots to communicate. In order to take co-operation to a higher level, the framework will need concepts such as role changes and set plays. Further, there is also the need for game statistics, which enable the modelling of the opponent team and could be the basis for a better choice of tactics, prior to and during the game. All these concepts will be considered in the future as possible extensions.

\section{References}

1. Röfer, T., Brunn, R., Czarnetzki, S., Dassler, M., Hebbel, M., Jüngel, M., Kerkhof, T., Nistico, W., Oberlies, T., Rohde, C., Spranger, M., Zarges, C.: Germanteam 2005 - the german national robocup team. In: RoboCup 2005: Robot Soccer World Cup IX, Springer Verlag (2006)

2. Mota, L., Reis, L.P., Burkhard, H.D.: Communication challenges raised by open co-operative teams in robocup. In: Encontro Científico do Festival Nacional de Robótica 2006. (2006)

3. Utz, H., Stulp, F., Muehlenfeld, A.: Sharing belief in teams of heterogenous robots. In Nardi, D., Riedmiller, M., Sammut, C., eds.: RoboCup-2004: The Eighth RoboCup Competitions and Conferences. Lecture Notes in Artificial Intelligence, Berlin, Springer Verlag (2004)

4. ISO: Guide to the expression of uncertainty in measurement. International Organization for Standardization (ISO). (1995)

5. Reis, L.P., Lau, N.: Coach unilang - a standard language for coaching a (robo) soccer team. In Birk, A., Coradeschi, S., Tadokoro, S., eds.: RoboCup-2001: Robot Soccer World Cup V. Volume 2377 of Lecture Notes in Artificial Intelligence., Berlin, Springer Verlag (2002) 183-192

6. Davin, J., Riley, P., Veloso, M.: CommLang: Communication for coachabe agents. In Nardi, D., Riedmiller, M., Sammut, C., eds.: RoboCup-2004: The Eighth RoboCup Competitions and Conferences. Lecture Notes in Artificial Intelligence, Berlin, Springer Verlag (2004)

7. FIPA: FIPA SL Content Language Specification. Foundation for Intelligent Physical Agents. (2002)

8. FIPA: FIPA Request Interaction Protocol Specification. Foundation for Intelligent Physical Agents. (2002) 